\title{
Effect of Lycium barbarum on Immune Function of Plateau Training Plain Middle and Long Distance Runners
}

\author{
Xiaoming Ma1, Rongyun Fan ${ }^{2,3 *}$, Fuhai Ma ${ }^{2,3}$ \\ ${ }^{1}$ Qinghai Nationalities University, Xining, China \\ ${ }^{2}$ Qinghai Research Institute of Sports Science, Key Laboratory of Highland Training and Research of General Administration of \\ Sports, Key Laboratory of Highland Sports Science of Qinghai, Xining, China \\ ${ }^{3}$ Research Institute of Plateau Science and Sustainable Development, Xining, China \\ Email: *592707294@qq.com
}

How to cite this paper: Ma, X. M., Fan, R. Y., \& Ma, F. H. (2020). Effect of Lycium barbarum on Immune Function of Plateau Training Plain Middle and Long Distance Runners. Advances in Physical Education, 10, 333-341.

https://doi.org/10.4236/ape.2020.104026

Received: August 17, 2020

Accepted: October 12, 2020

Published: October 15, 2020

Copyright ( 2020 by author(s) and Scientific Research Publishing Inc. This work is licensed under the Creative Commons Attribution International License (CC BY 4.0).

http://creativecommons.org/licenses/by/4.0/

\begin{abstract}
During the altitude training at $2366 \mathrm{~m}$ altitude, the immunoglobulin and IL-6 of male athletes of middle and long distance running in the plain were measured. Taking Qinghai Lycium barbarum can increase immunoglobulin, CD8+ T lymphocyte and NK cell, reduce the decline of IL-6, CD3+, CD4+ T lymphocyte and $\mathrm{CD} 4+/ \mathrm{CD} 8+$, which is beneficial to improve the immune ability of athletes and ensure the effect of altitude training.
\end{abstract}

\section{Keywords}

Lycium barbarum, Altitude Training, Athletes, Immune Function

\section{Introduction}

The influence of exercise on immune system function is restricted by many external factors. Heavy load and high-intensity sports training can inhibit immune function and improve the body's susceptibility to diseases, thus affecting the health level and sports ability of athletes (Wang \& Gao, 2019). Hypoxic environment can cause the disorder and decline of immune function (Li et al., 2017; $\mathrm{Pu}$ et al., 2018), and the damage of immune function under hypoxic condition is more serious than that of pure hypoxic environment or simple exercise training (Jin, Hu, \& Jin, 2015), while the dual stimulation of hypoxic environment and exercise load during high altitude training will cause more complex impact on immune system (Gao et al., 2018). Therefore, it is very important to take effective measures to prevent the decline of athletes' immune function during altitude training to ensure the training effect. 
Lycium barbarum not only has rich biological activities, but also has no toxic and side effects. Its pharmacological effect is to affect the immune function of human body. It has the effects of hematopoiesis, enhancing immunity, antioxidant, anti hypoxia, anti-fatigue and so on (Wang et al., 2017). Studies have confirmed that Lycium barbarum polysaccharide can effectively enhance the humoral immune function and anti-fatigue ability of sub-health mice (Hao et al., 2015), Lycium barbarum can significantly improve the anti-hypoxia and anti-fatigue ability of mice (Zhang, 2020), and wolfberry juice has a very obvious role in improving the body's adaptability to exercise load, delaying the generation of fatigue and accelerating the elimination of fatigue ( $\mathrm{Hu}$ et al., 2008). However, there are few reports on the effects of Lycium barbarum juice on the immune function of human body, especially athletes.

Thanks to the unique climate conditions of Qinghai Tibet Plateau, Qinghai Lycium barbarum fresh fruit has rich nutritional value (Yang et al., 2012), rich in vitamins and amino acids, especially in the medical and health care functions of Lycium barbarum polysaccharide content as high as $8.3 \%$, and is the best quality Lycium barbarum in China (Cui et al., 2020). Lycium barbarum juice is the juice product obtained by physical methods such as squeezing, centrifugation and extraction with fresh Lycium barbarum fruit as raw material. Pure Lycium barbarum juice is a new type of zero addition, non-concentrated and reduced Lycium barbarum juice, which fully retains the main functional components and nutritional elements of fresh Lycium barbarum (Weng et al., 2016). This study was conducted to observe and summarize the effects of Lycium barbarum L. on the immunity of athletes during altitude training in plain men's middle-distance runner, thus, the research and application of altitude adaptation and altitude training can be referred to Lycium barbarum.

\section{Research Methods}

\subsection{Research Object}

According to the matching principle of age and sports level, 18 elite male middle and long distance runners were selected and divided into the experimental group and the control group according to the matching principle of age and sports level. Among them, the experimental group had 10 athletes, including 3 elite athletes, 4 first-class athletes and 3 second-class athletes, with an average age of $21.00 \pm 3.71$ years, an average of $116.0 \pm 225.6 \mathrm{~m}$ in the birthplace, and $5.20 \pm$ 4.49 years of professional training The average age of the control group was $19.38 \pm 3.11$ years, the average altitude of birth was $143.8 \pm 173.8 \mathrm{~m}$, and the professional training period was $5.50 \pm 4.34$ years.

\subsection{Research Plan}

The control group and the experimental group received altitude training for 30 days from the plain to the altitude of $2366 \mathrm{~m}$, during which the two groups received the same content and load intensity of exercise training. The experi- 
mental group took 2 pieces of pure Lycium barbarum juice before going to bed every night, while the control group did not drink it. The results of pony test showed that every $100 \mathrm{ml}$ of Lycium barbarum juice contained 360 - $440 \mathrm{mg}$ of LBP, and it did not contain any stimulants as determined by China Anti Doping center.

\subsection{Research Indicators and Methods}

The number of immune cells and lymphocytes plays an important role in monitoring the changes of immune function under hypoxia training (Li \& Lin, 2018). In this study, the indexes of immunoglobulin (Ig), interleukin (IL), T lymphocyte subsets and natural kill cell (NK) in blood were detected and compared.

On the first day (basic value), the 14th day (intermediate value) and the 28th day (end value) of altitude training, $3 \mathrm{ml}$ of elbow venous blood was collected for detection of immunoglobulin (IGA, IgG, IgM) and interleukin-6 (IL-6) by EDTA-K2 anticoagulant, and $4 \mathrm{ml}$ of elbow vein blood was taken from common non-anticoagulant blood collection vessel, centrifuged at $3000 \mathrm{r} / \mathrm{min}$ for $15 \mathrm{~min}$ T lymphocyte subsets (CD3+, CD4+, CD8+) and NK cell subsets (CD3 - CD16+ or $\mathrm{CD} 56+$ ) were measured. Immunoglobulin was detected by turbidimetric method in Cobas 8000 c702, IL-6 in Siemens IMMULITE 1000 chemiluminescence analyzer, serum $\mathrm{T}$ lymphocyte subsets and NK lymphocytes were detected by flow cytometry in American Backman cyto flex flow cytometry. Spss20.0 was used to analyze the test data. Paired t-test, independent sample t-test and one-way ANOVA were used to analyze the changes of each index in different stages of the same group and between groups.

\section{Research Results}

\subsection{Immunoglobulin}

In the IgA experimental group, the intermediate value increased by $13.8 \%$, and the end value was still higher than the basic value by $5.7 \%$, while the intermediate value of the control group decreased by $22.4 \%$, and the end value returned to the basic level; the intermediate value of the IgG experimental group increased by $3.6 \%$, and the end value was close to the basic value, while the intermediate value of the control group decreased by $14.9 \%$, and the end value was still lower than the basic value by $9 \%$; after the intermediate value of IgM experimental group decreased (8.7\%), the end value returned to the basic level The control group showed a continuous decrease $(6.1 \%$ - 10.5\%) during altitude training (Table 1).

\subsection{Interleukin 6}

During altitude training, the serum IL-6 of the two groups showed a downward trend. Compared with the basic value, the mid-term value and the end value of the experimental group decreased by $17 \%$, while the control group decreased by $32 \%$ - 37\% (Table 1). 
Table 1. Statistical results of immunoglobulin and interleukin-6 of plain athletes during altitude training (mean $\pm \mathrm{SD}$ ).

\begin{tabular}{|c|c|c|c|c|c|}
\hline Classification & Index & Grouping & $\begin{array}{l}\text { Base } \\
\text { value }\end{array}$ & $\begin{array}{c}\text { Intermediate } \\
\text { value }\end{array}$ & $\begin{array}{l}\text { Terminal } \\
\text { value }\end{array}$ \\
\hline \multirow{6}{*}{ Immunoglobulin } & \multirow{2}{*}{$\operatorname{IgA}(\mathrm{g} / \mathrm{L})$} & experience group & $1.98 \pm 0.53$ & $2.26 \pm 0.85$ & $2.10 \pm 0.73$ \\
\hline & & control group & $1.76 \pm 0.86$ & $1.37 \pm 0.41$ & $1.76 \pm 0.99$ \\
\hline & \multirow{2}{*}{$\operatorname{IgG}(\mathrm{g} / \mathrm{L})$} & experience group & $9.02 \pm 1.69$ & $9.35 \pm 1.99$ & $8.99 \pm 2.36$ \\
\hline & & control group & $9.02 \pm 1.70$ & $7.68 \pm 0.99$ & $8.17 \pm 1.77$ \\
\hline & \multirow{2}{*}{$\operatorname{IgM}(\mathrm{g} / \mathrm{L})$} & experience group & $0.96 \pm 0.35$ & $0.87 \pm 0.13$ & $0.96 \pm 0.23$ \\
\hline & & control group & $1.09 \pm 0.22$ & $1.02 \pm 0.22$ & $0.98 \pm 0.07$ \\
\hline \multirow{2}{*}{ Interleukin } & \multirow{2}{*}{ IL-6 (pg/mL) } & experience group & $1.52 \pm 1.02$ & $1.25 \pm 0.82$ & $1.26 \pm 0.54$ \\
\hline & & control group & $1.87 \pm 1.42$ & $1.17 \pm 0.82$ & $1.26 \pm 0.24$ \\
\hline
\end{tabular}

\subsection{T Lymphocytes}

During high altitude training, except for the experimental group, the medium-term value of CD8+ increased by $11.9 \%$, and the later end value decreased to the basic value in the experimental group, the mid-term value of $\mathrm{CD} 3+\mathrm{de}-$ creased by $3.7 \%$, the terminal value decreased by $9.4 \%$, the mid-term and terminal value of $\mathrm{CD} 4+$ decreased by $17 \%-19 \%$, the mid-term value of CD4+/CD $8+$ decreased by $15.5 \%(P<0.05)$, and the end-stage value decreased by $6.8 \%$; in the control group, the mid-term value of $\mathrm{CD} 3+$ decreased by $13.6 \%$, the end-stage value decreased by $7.9 \%$, CD $4+$ decreased by $13 \%-18 \%$, CD $8+$ by $5 \%-11 \%$, and the median CD4+/CD8+ value decreased by $12.9 \%$ and the end-stage value decreased by $10.7 \%$ (Table 2). Compared with the control group, the decrease of $\mathrm{CD} 3+$ and $\mathrm{CD} 4+/ \mathrm{CD} 8+$ in the experimental group was lower, and CD8+ showed an increasing trend.

\subsection{NK Cells}

NK cells in the experimental group showed a continuously increasing trend (Table 2). Compared with the basic value, the end-stage value increased by $49.8 \%(P<0.05)$, while that in the control group increased by $22.3 \%$, which was lower than that in the experimental group.

\section{Analysis and Discussion}

The effects of different exercise intensities on immunoglobulin are different. Moderate and regular exercise can increase the contents of $\operatorname{IgA}$, IgM and IgG, and enhance the immune function of the body. However, the contents of IgA, IgM and IgG significantly decrease during long-term and high-intensity exercise, resulting in the decrease of immune function of the body (Xing \& Li, 2007). During winter training, the humoral immune indexes such as serum immunoglobulin of high-level kayak athletes are relatively lower, and their immune ability is faced with great risk. Rhinitis, influenza and other diseases without obvious 
Table 2. Statistical results of T lymphocyte and NK cell of plain athletes during altitude training (mean $\pm \mathrm{SD})$.

\begin{tabular}{|c|c|c|c|c|c|}
\hline Classification & Index & Grouping & $\begin{array}{l}\text { Base } \\
\text { value }\end{array}$ & $\begin{array}{c}\text { Intermediate } \\
\text { value }\end{array}$ & $\begin{array}{l}\text { Terminal } \\
\text { value }\end{array}$ \\
\hline \multirow{8}{*}{ T lymphocyte } & \multirow{2}{*}{$\mathrm{CD} 3+(\%)$} & Experience group & $65.10 \pm 12.97$ & $62.67 \pm 10.21$ & $59.00 \pm 9.94$ \\
\hline & & control group & $64.13 \pm 8.98$ & $55.40 \pm 9.81$ & $59.00 \pm 10.43$ \\
\hline & \multirow{2}{*}{ CD4+ (\%) } & experience group & $33.20 \pm 7.58$ & $27.50 \pm 3.89$ & $26.89 \pm 4.17^{\star}$ \\
\hline & & control group & $36.25 \pm 7.49$ & $29.60 \pm 5.03$ & $31.50 \pm 4.76$ \\
\hline & \multirow{2}{*}{ CD8+ (\%) } & experience group & $29.5 \pm 7.82$ & $33.00 \pm 8.46$ & $29.67 \pm 8.28$ \\
\hline & & control group & $24.63 \pm 5.04$ & $22.00 \pm 6.82$ & $23.50 \pm 6.83$ \\
\hline & \multirow{2}{*}{ CD4+/CD8+ } & experience group & $1.03 \pm 0.15$ & $0.87 \pm 0.23^{*}$ & $0.96 \pm 0.23$ \\
\hline & & control group & $1.40 \pm 0.40$ & $1.22 \pm 0.33$ & $1.25 \pm 0.28$ \\
\hline \multirow{2}{*}{ NK cells } & \multirow{2}{*}{$\begin{array}{l}\text { CD3 - CD16+ } \\
\text { or CD56+ (\%) }\end{array}$} & experience group & $20.40 \pm 11.38$ & $27.33 \pm 8.19$ & $30.56 \pm 8.69^{\star}$ \\
\hline & & control group & $19.63 \pm 8.83$ & $27.75 \pm 6.90$ & $24.00 \pm 5.00$ \\
\hline
\end{tabular}

Note: compared with the basic value, ${ }^{\star}$ means significant difference $(P<0.05)$, and ${ }^{* *}$ means very significant difference $(P<0.01)$.

pathogenic factors occur frequently (Li \& Zhang, 2014). High altitude or hypoxia training can have a certain impact on plasma immunoglobulin (Tiolier et al., 2005). Hypoxia can cause salivary IgA to decrease. Gao Binghong et al. found that living at high altitude and training low oxygen for 4 weeks can inhibit the immune function of elite female rowers (Gao \& Chen, 2014). Reasonable supplement of nutrients is conducive to promoting the recovery of immune function. The supplementation of LBP in high-intensity endurance exercise can effectively prevent the decline of immune function and help maintain the immune system (Liu, Pan, \& Wang, 2011). During Qinghai plateau training, IgA, IgG and IgM of the control group showed different degrees of decline, which indicated that the immune function of plain athletes decreased due to the dual stimulation of exercise training load and hypoxia in high altitude environment, while the immunoglobulin IgA, IgG and IgM in the experimental group increased slightly, and the immune function of the body was improved to some extent In order to improve the athletes' immunoglobulin function, effectively alleviate the exercise-induced immunosuppression.

The main role of IL-6 is to promote the proliferation and differentiation of B cells and secrete antibodies, which is conducive to the body to resist pathogenic microorganisms, and has obvious anti-inflammatory effect (Zhao, 2005). Studies have found that IL-6 plays an important regulatory role in skeletal muscle metabolism (Pedersen, Steensberg, \& Schjerling, 2001). When skeletal muscle intake of glucose increases over a long period of time, IL-6 in the body can play an important role in regulating liver metabolism, so as to maintain the stability of blood glucose level during exercise; at the same time, IL-6 also participates in promoting the decomposition of fat and other metabolic pathways (Stouthard et al., 1996), which can promote the decomposition of fatty acids and improve the 
level of fatty acids The amount of free fatty acids available to meet the energy needs of exercise muscles. In addition, IL-6 plays an important role in promoting angiogenesis, inducing and regulating erythropoietin (EPO) production and inducing vascular endothelial growth factor (VEGF) (Ma, 2012; Zhu, Wu, \& Geng, 2004). Compared with the control group, the decrease of IL-6 in the experimental group was lower than that in the control group, which suggested that during the plateau training, taking Lycium barbarum juice could alleviate the decline of IL-6 in plain athletes, which was beneficial to maintain blood glucose level and promote fat catabolism, as well as the formation of VEGF and EPO regulatory factors, so as to improve sports endurance and anti hypoxia ability.

The research confirmed that (Gao, Wu, \& Li, 2011) after long-term heavy load training, the content of $\mathrm{CD} 3+, \mathrm{CD} 4+, \mathrm{CD} 8+$ decreased, and the ratio of $\mathrm{CD} 4+/ \mathrm{CD} 8+$ decreased, which led to the disorder of immune regulation function. Hypoxic environment exposure or hypoxic training can lead to changes in $\mathrm{T}$ lymphocytes. After acute and chronic hypoxia exposure at $5050 \mathrm{~m}$ altitude, CD3+ and CD4+ T lymphocytes decreased (Facco et al., 2005), resulting in the damage of THL/Th2 immune balance homeostasis regulation and increased risk of infection. During hypoxic training, the level of CD3+ and CD4+/CD8+ decreased by $4.13 \%$ and $2.4 \%$, respectively (Wang \& Gao, 2014). During altitude training, the combined effect of hypoxia and exercise can reduce the immune indexes of athletes. CD8+ is more sensitive to low-pressure hypoxia double stimulation (Wang \& Gao, 2019). Generally, the decrease of CD4+/CD8+ ratio of athletes is regarded as a sign of low immune function.

In this study, the levels of CD3+, CD4+, CD8+ and CD4+/CD8+ in the experimental group and the control group showed a downward trend, in which CD3+ decreased by $3.7 \%-13.6 \%$, CD4+ decreased by $13 \%-18 \%$, and CD $4+/ C D 8+$ decreased by $6.8 \%-15.5 \%$. However, the decrease of CD3+ and CD4+/CD $8+$ in the experimental group was lower than that in the control group, and CD8+ showed an increasing trend. The experiment shows that (Li, 2008) LBP supplementation can significantly increase the $\mathrm{CD} 3+, \mathrm{CD} 4+, \mathrm{CD} 4+/ \mathrm{CD} 8+$ ratio, IgA, IgG, cellular immunity and humoral immunity of volleyball players who have been engaged in intensive training for a long time. In this study, athletes taking pure Lycium barbarum juice during high altitude training can effectively alleviate the imbalance of immune function caused by exercise and hypoxia, reduce the decrease range of $\mathrm{CD} 3+$, increase $\mathrm{CD} 8+$, and appropriately reduce the imbalance of $\mathrm{CD} 4+/ \mathrm{CD} 8+$, which is beneficial to the middle and long term of plain Runners can improve the immune function and the ability to adapt to hypoxia.

In the process of exercise, NK cells are the most significantly changed lymphocytes. Various acute exercises can increase the number and activity of NK cells, while long-term and high-intensity exercise will cause the decrease of NK cell count and function (Li, 2016). In addition, hypoxia can affect NK cells. Gao found that after 4 weeks of HiLo hypoxia training (Gao, Wu, \& Sheng, 2014), the NK cells of female rowers decreased significantly and lasted for a long time. Studies have confirmed that (Gao, Wu, \& Li, 2011), heavy load training can cause 
the number of NK cells of wrestlers to decrease, acupuncture pretreatment can improve NK cell activity, thus changing the immune function disorder of the body, and has positive significance in fighting fatigue. Liu (Liu, Pan, \& Wang, 2011) and other experiments showed that LBP supplementation in high-intensity endurance exercise can increase the number of NK cells and effectively prevent the decline of immune function. Peng (Peng et al., 2019) found that after drinking pure Lycium barbarum juice for 32 days, Th cells, NK cells and serum immunoglobulin IgA and IgG in adult men of different age groups were increased. It is believed that drinking pure Lycium barbarum juice has a good positive regulatory effect on immune function of the body. In this study, the increase of NK cells in the experimental group of athletes taking medlar juice during altitude training was greater than that of the control group $(P<0.05)$. It is suggested that Lycium barbarum juice can play the role of immune enhancer. By mobilizing the reserve function of thymus of central immune organs, more mature NK lymphocytes are released to the peripheral to make up for the deficiency and play a role in hypoxia exposure, which can fully regulate the motivation The body adapts to the sudden change of oxygen concentration as soon as possible, so as to minimize the influence and damage of hypoxia on the body, so as to realize the protective effect on the body (Tian et al., 2010).

\section{Research Conclusion}

During the altitude training at $2366 \mathrm{~m}$ altitude, the level of immunoglobulin IgA, IgG, IgM, IL-6, T lymphoid CD3+, CD4+ and CD4+/CD8+ decreased in varying degrees. The NK cells increased. Taking Lycium barbarum juice can help to increase IgA, IgG, CD8+, NK, and reduce the levels of IL-6, CD3+, CD4+/CD8+ in plain male middle- and long-distance runners, which is conducive to improving the athletes' immune ability.

\section{Fund}

This work is supported by basic research project of science and technology application in Qinghai (No. 2019-ZJ-7077, 2019-ZJ-7010) and Key R \& D and transformation projects of science and technology in Qinghai (No. 2019-SF-126, 2019-HZ-810).

\section{Conflicts of Interest}

The authors declare no conflicts of interest regarding the publication of this paper.

\section{References}

Cui, Z. J., Liu, F. L., Zhang, Q. L. et al. (2020). Comparative Analysis of Lycium barbarum Polysaccharide Content in Lycium barbarum L. from Different Habitats. Western China Medical Journal, 35, 59-61.

Facco, M., Zilli, C., Siviero, M. et al. (2005). Modulation of Immune Response by the Acute and Chronic Exposure to High Altitude. Medicine \& Science in Sports \& 
Exercise, 37, 768-774. https://doi.org/10.1249/01.MSS.0000162688.54089.CE

Gao, B. H., \& Chen, P. J. (2014). Effects of Living High and Training Low for 4 Weeks on Immune Function of Female Rowers. Chinese Journal of Sports Medicine, 33, 404-412.

Gao, H., Li, T., Gao, B. H. et al. (2018). Immune and Inflammatory Response Characteristics of 3200 mhihilo and Its Influence on Aerobic Exercise Ability. Sports Science, 38, 54-60.

Gao, M., Wu, Y., \& Li, G. Q. (2011). Effects of Moxibustion on T Lymphocyte Subsets of Male Middle and Long Distance Runners during Heavy Load Training. Chinese Journal of Sports Medicine, 30, 997-1001.

Gao, M., Wu, Y., \& Sheng, Z. T. (2014). Effects of Acupuncture Pretreatment on NK Cells and T Lymphocyte Subsets of Wrestlers during Heavy Load Training. Shanghai Institute of Physical Education, 38, 67-72.

Hao, W. L., Chen, Z. B., Zhao, R. et al. (2015). Effects of Lycium barbarum Polysaccharides on Immune Function and Anti Fatigue Effect in Sub-Health Mice. Chinese Journal of Biological Products, 28, 693-697.

Hu, J. W., Fan Q. S., Xiong, W. et al. (2008). Study on Anti Fatigue Effect of Chinese Wolfberry Juice. Jiangxi Food Industry, 2, 47-49.

Jin, Q. G., Hu, Y. J., \& Jin, A. N. (2015). Effects of Different Modes of Hypoxic Exercise Training on Intestinal Humoral Immune Function in Rats. Chinese Journal of Sports Medicine, 34, 764-769.

Li, C., Liu, T., Bazhin, A. V. et al. (2017). The Sabotaging Role of Myeloid Cells in Anti-Angiogenic Therapy: Coordination of Angiogenesis and Immune Suppression by Hypoxia. Journal of Cellular Physiology, 232, 2312-2322. https://doi.org/10.1002/jcp.25726

Li, J. Y. (2016). Research on the Changes of NK and NKT Cells of Sanda Athletes after Intensive Training. Shandong Sports Science and Technology, 38, 60-62.

Li, L. (2008). Effects of LBP Supplementation on Immune Function of Volleyball Players. Journal of Beijing Sport University, 31, 1084-1086.

Li, M., \& Zhang, L. (2014). Characteristics of Serum Immunoglobulin and Complement C3 and C4 of Kayak Athletes during Winter Training. Sports Research, 35, 62-66.

Li, Z. G., \& Lin, W. C. (2018). Visualization Analysis of the Research Status of Hypoxia Training in China. Sports Research, 39, 56-65.

Liu, X. N., Pan, C. G., \& Wang, Q. (2011). Effects of Lycium barbarum Polysaccharides on Immune Function after High Intensity Endurance Exercise. Journal of Ningxia Medical University, 33, 801-802.

Ma, X. F. (2012). Altitude Exercise and Immune Stress. Journal of Plateau Medicine, 22, 58-61.

Pedersen, B. K., Steensberg, A., \& Schjerling, P. (2001). Muscle-Derived Inerleukin-6: Possible Biological Effects. The Journal of Physiology, 536, 329-337. https://doi.org/10.1111/j.1469-7793.2001.0329c.xd

Peng, Y. Q., Xu, G. Q., Weng, X. Q. et al. (2019). Effects of Pure Lycium barbarum Juice on Immune Function of Healthy Adults Aged 31-65. Guangdong Medical Journal, 40, 732-735.

Pu, X. Y., Hu, F. J., Li, J. D. et al. (2018). Effects of High Altitude Hypoxia on Cytotoxicity and Secretion of IL- 6 and TNF- $\alpha$ by Macrophages in Mice. Chinese Journal of Immunology, 34, 344-348.

Stouthard, J. M. et al. (1996). Interleukin-6 Enhances Glucose Transport in 3 T3-L1 
Adipocytes. Biochemical Biophysical Res Communications, 220, 241-245. https://doi.org/10.1006/bbrc.1996.0389

Tian, Y. M., Nie, H. J., Liu, J. Y. et al. (2010). Study on High Altitude Hypoxia Immune Injury and Its Intervention Measures. Chinese Journal of Applied Physiology, 26, 404-409.

Tiolier, E., Schmit, L., Burnat, P. et al. (2005). Living High-Training Low Altitude Training: Effects on Mucosal Immunity. European Journal of Applied Physiology, 94, 298-304. https://doi.org/10.1007/s00421-005-1317-4

Wang, F., Ding, C. C., Li, W. X. et al. (2017). To Study the Effect of Lycium barbarum on Anti Hypoxia and Anti Fatigue Ability in Mice. Journal of Integrated Chinese and Western Medicine, 34, 167-168.

Wang, X., \& Gao, B. H. (2014). Effects of 3-Week High Living Low Training on Erythrocyte Immunity and Immune System of Elite Rowers. Chinese Journal of Sports Medicine, 33, 1054-1059.

Wang, X., \& Gao, B. H. (2019). Research Progress on the Influence of Hypoxic Environment and Sports Training on Erythrocyte Immune Function. Sports Research, 40, 93-98.

Weng, X. Q., Lin, J. R., Xu, G. Q. et al. (2016). Effects of Medlar Juice on Serum Testosterone and Cortisol Levels of Male College Students during Incremental Exercise. Chinese Journal of Sports Medicine, 35, 344-348.

Xing, L. M., \& Li, J. (2007). Effects of Exercise on Serum Immunoglobulin IgA, IgM and IgG. Journal of Jilin Institute of Physical Education, 23, 81-82.

Yang, R. M., Jing, N. H., Wang, H. L. et al. (2012). Analysis and Evaluation of Nutritional Composition and Bioactive Composition in Chinese Wolfberry (Lycium barbarum L.) Fruits from Different Regions of Qinghai Province. Food Science, 33, 265-269.

Zhang, Q. W. (2020). Development of Maosuanzi Lycium barbarum Liquid Nutritional Beverage and Its Anti Fatigue Effect on Sports. Food Research and Development, 41, 141-145.

Zhao, K. W. (2005). Research Progress on the Effects of Exercise on Interleukin-1, Interleukin-2 and Interleukin-6. Journal of Capital Institute of Physical Education, 17, $43-45,67$.

Zhu, H. H., Wu, H. F., \& Geng, P. L. (2004). Changes of IL-1 and IL-6 Contents under Hypoxia and Their Significance. Journal of Qinghai Medical College, 25, 94-96. 